\title{
ACKNOWLEDGMENT
}

\section{Acknowledgment of the Scientific Contributors}

The Scientific Program Committee of the 16th International Congress of Cytology wishes to thank all contributors to the conference and acknowledge the high standard of the work presented in this publication. In addition, the committee wishes to thank all invited lecturers and contributors to the various Satellite Symposia. We offer our congratulations to those receiving awards from the International Academy of Cytology.

The Committee wishes to inform readers that all abstracts were submitted to peer review by members of the Canadian Society of Cytology. Using a standard protocol, each abstract was graded by 2 independent reviewers with special expertise in the particular area addressed by the abstract. The clinical or scientific importance of the work was scored 0 through $5[0, \mathrm{Re}-$ ject; 1, Poor; 2, Fair; 3, Good; 4, Very Good; 5, Excellent]. In addition, reviewers were asked to indicate whether an abstract provided significant new information or would be likely to stimulate discussion or further research. In cases in which a marked discrepancy between the 2 reviewers was noted, a third reviewer provided an independent additional opinion.

The abstracts were ranked in order of excellence, and selections for oral presentations were made from cases receiving a score of 3.5 or more out of 5 . Most abstracts accepted for oral platform presentations scored 4 or 5 . Initially, 72 oral platform presentations and 187 poster presentations were accepted, although some works have subsequently been withdrawn by the authors. Seven abstracts were rejected by the reviewers.

Assessment of workshop submissions was particularly difficult, with 37 applications made for 12 workshops. The workshop submissions were similarly graded and then ranked. In addition, reviewers were asked to state whether the workshop covered a novel topic or offered a novel approach to a well-known topic. Some excellent workshops were not accepted because they had been offered at several other conferences over a period of several years. The organizers regret the necessary limitations of space, time and number of microscopes available for workshops.

The Scientific Program Committee wishes to thank the Editors of Acta Cytologica for their assistance and for making possible the publication of this conference supplement. We thank the following reviewers for their expert contribution to this publication: Drs. Manon Auger, Diponkar Banerjee, Terry Colgan, Maire Duggan, Dan Fontaine, Malcolm Hayes, Linda Kapusta, Moosa Khalil, Karim Khetani, Bryan Knight, Jasenka Matisic, Calum MaCauley, Meg McLachlin, Tom Thompson, Dirk van NieKirk, Ranjit Waghray and Michele Weir. Editorial assistance was provided by Michelle Mori of Venue West Conference Services.

Bryan Knight, M.B., Ch.B., Ph.D., F.I.A.C. Abstract Editor 